\title{
Characteristics of coronary microcirculatory function in patients with Takotsubo syndrome
}

\author{
Giuseppina Novo ${ }^{1}$, Angelo Quagliana ${ }^{1}$, Dario Buccheri ${ }^{2}$, Salvatore Rizzo ${ }^{1}$, Salvatore Giambanco ${ }^{1,3}$, \\ Francesco Giambanco ${ }^{3}$, Salvatore Evola ${ }^{1}$, Giovanna Geraci ${ }^{4}$, Salvatore Novo $^{1}$ \\ ${ }^{1}$ Chair and Division of Cardiology, University of Palermo, Palermo, Italy; ${ }^{2}$ Interventional Cardiology, San Giovanni di Dio Hospital, Agrigento, \\ Italy; ${ }^{3}$ Division of Cardiology, Ingrassia Hospital, Palermo, Italy; ${ }^{4}$ Division of Cardiology, Cervello Hospital, Palermo, Italy \\ Contributions: (I) Conception and design: G Novo; (II) Administrative support: S Novo, G Geraci; (III) Provision of study materials or patients: D \\ Buccheri, S Giambanco; (IV) Collection and assembly of data: S Rizzo, F Giambanco; (V) Data analysis and interpretation: A Quagliana, S Evola; (VI) \\ Manuscript writing: All authors; (VII) Final approval of manuscript: All authors. \\ Correspondence to: Dario Buccheri, MD. Interventional Cardiology, San Giovanni di Dio Hospital, Agrigento, Italy. Email: dariobuccheri@gmail.com; \\ Giuseppina Novo, MD, PhD. Chair of Cardiology, University of Palermo, Via Del Vespro 129, 90127 Palermo, Italy. Email: giuseppina.novo@unipa.it.
}

Background: Takotsubo syndrome (TS) is a recently described cardiac syndrome whose pathogenesis is still unclear. We investigated the characteristics of coronary microcirculatory function in patients with TS through the analysis of the TIMI frame count (TFC) compared to normal subjects and with to subjects with microvascular angina (MA).

Methods: We enrolled 71 TS patients ( $F: M=69: 2$, mean age of $65.27 \pm 9.53$ years), 70 controls ( $F: M=34: 36$, mean age of $56.63 \pm 13.5$ years) and 71 patients with $M A,(F: M=69: 2$, mean age of $65.9 \pm 9.2$ years). The assessment of the microcirculation was carried out through the TFC.

Results: microcirculation was significantly altered in patients with TS compared with healthy controls [left anterior descending coronary artery (LAD) $25.16 \pm 6.91$ vs. 17.30 $\pm 3.76, \mathrm{P}<0.001$; circumflex artery (CX) $25.48 \pm 6.10$ vs. $17.05 \pm 4.60, \mathrm{P}<0.001$; right coronary artery $(\mathrm{RCA}) 26.43 \pm 8.95$ vs. $15.74 \pm 4.27, \mathrm{P}<0.001$, average TFC in TS $25.70 \pm 5.34$ vs. $16,70 \pm 3.26, \mathrm{P}<0.001)$. A TFC $>20$ frames was able to discriminate TS patients from controls with a specificity of $88.57 \%$ and sensitivity of $85.92 \%$ (AUC $0.927, \mathrm{P}<0.0001$ ). Microvascular dysfunction was diffuse in TS as well as in MA and slightly more severe in this last (mean TFC in MA $28.25 \pm 9.3$ vs. $25.7 \pm 5.34$ in TS, $\mathrm{P}<0.046)$.

Conclusions: Coronary microcirculation in TS patients is diffuse and milder compared to MA patients. Cut-off values $>20$ frames discriminate between patients with normal microcirculation and patients with TS.

Keywords: Takotsubo; microvascular angina (MA); microvascular function; TIMI frame count (TFC)

Submitted Jan 14, 2017. Accepted for publication Oct 16, 2017.

doi: $10.21037 /$ jtd.2017.10.93

View this article at: http://dx.doi.org/10.21037/jtd.2017.10.93

\section{Introduction}

Takotsubo syndrome (TS) is an infrequent syndrome characterized by systolic dysfunction that usually gives a circumferential involvement of the basal and apical segments of the left ventricle (1-3). It is frequently related to acute physical and psychological stress and it is generally reversible (1). From a clinical standpoint, it strongly mimics an acute coronary syndrome (chest pain at rest and/or dyspnea), however coronary angiography reveals the absence of obstructive coronary artery disease or acute plaque rupture (1,3-5). The syndrome has found its own clinical and diagnostic identity only in recent years and it was included by the American Heart Association (AHA) in the classification of cardiomyopathies, as acquired cardiomyopathy (2). More recently the position paper of the ESC suggested using the term "syndrome" instead of cardiomyopathy because the full recovery of patients and 
Table 1 Clinical characteristics of patients with Takotsubo syndrome and microvascular angina

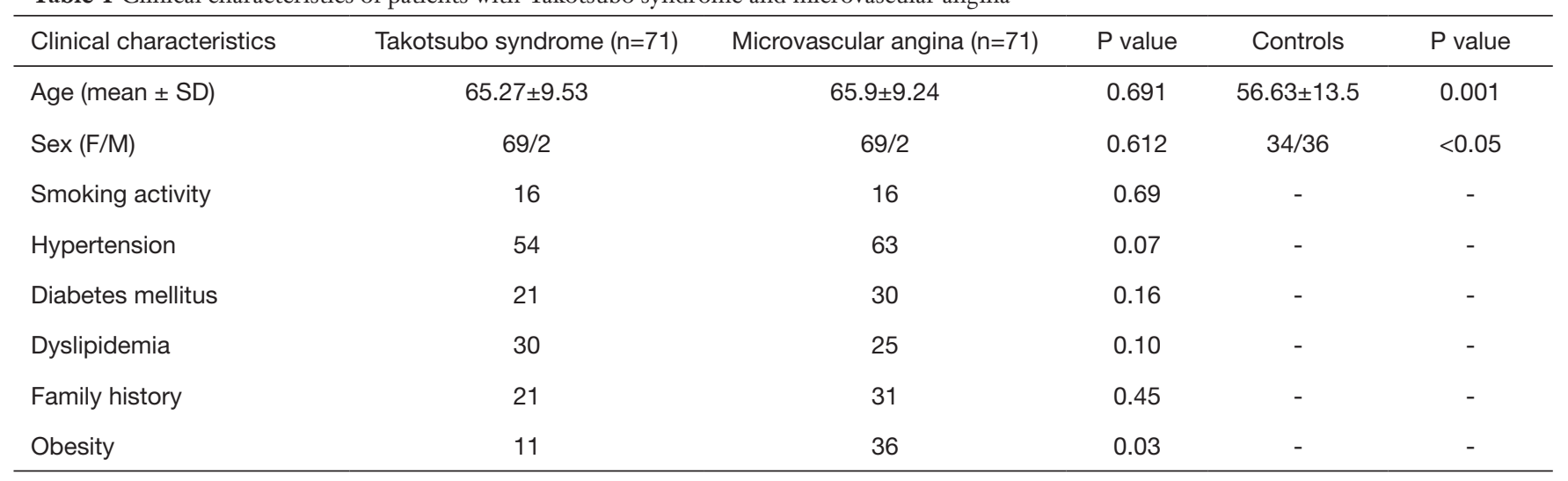

the low rate of major adverse cardiac events at follow-up in reported series strongly suggest that TS is different from the primary cardiomyopathies (6).

It was first described in the early 90 s in Japan and this had initially suggested that racial and genetic factors could be involved (7). Thereafter, it has been reported with increasing incidence in non-Asian populations, including the United States (8) and Europe (9-10). The term 'Takotsubo' derives from the name of the Japanese trap used by fishermen to catch octopuses. This resembles the shape taken by the left ventricle, due to typical wall motion abnormalities involving the apex and the mid segments (1). For this evidence, the syndrome is also called: 'left ventricular apical ballooning'. The pathogenesis of TS is still uncertain. The proposed hypothesis in the literature is an epicardial multivessel coronary spasm, neurogenic myocardial stunning, catecholaminergic cardiotoxicity, and microvascular dysfunction, being the last two the most accredited. The aim of our study was to investigate the characteristics of coronary microcirculatory function in patients with TS, analyzed by TIMI frame count (TFC), in comparison with patients with microvascular angina (MA) and controls.

\section{Methods}

In this multicenter study, we retrospectively selected patients with a diagnosis of TS and we have compared them with a group of control subjects without cardiovascular risk factors, coronary artery epicardial disease and microvascular dysfunction at coronary angiography and with a group of patients suffering from MA. The diagnosis of TS has been made in agreement with the criteria proposed by the Mayo Clinic group (11).

Patients without coronary artery disease were recruited among patients undergoing coronary angiography before scheduled non-coronary cardiac surgery.

The diagnosis of MA was made up if patients had chest pain, ECG changes during the stress test and coronary arteries free from significant stenosis, but a demonstration of microvascular dysfunction at the coronary angiography. Patients with MA were matched by sex and age with TS.

All patients at admission were subjected to careful anamnestic evaluation and cardiovascular risk factors (family history, smoking activity, hypertension, dyslipidemia, diabetes mellitus type 1 and 2, obesity) and history of cardiovascular and cerebrovascular diseases (previous angina, acute myocardial infarction, PCI or stroke/TIA) were recorded. A venous blood sample was collected, on admission, for the evaluation of routine biochemical parameters and the determination of cardiac I troponin (Vitros, Ortho-Clinical Diagnostics). Moreover, patients underwent 12 lead electrocardiogram, transthoracic echocardiogram, and coronary angiography. Left ventricular function was assessed at admission and before discharge by measuring the left ventricular ejection fraction (LVEF), using the biplane Simpson's method. Coronary angiography was performed by selective catheterization, according to Seldinger's technique, cannulating a peripheral artery (femoral or radial). Microcirculation was tested by analyzing the TFC according to the Gibson's technique, as the number of frames required by the contrast medium to reach a standard reference point of a distal coronary vessel (12).

Due to the longer length of LAD, compared to circumflex and right coronary artery, the TFC values were divided by the conventional correction factor for LAD: 1.7 (12).

The TFC in the population of patients with TS was compared with that of controls and with patients with 
Table 2 TFC on LAD, CX and RCA and average TFC for all three vessels in patients with TS, controls and MA

\begin{tabular}{|c|c|c|c|c|c|}
\hline TFC & TS & Controls & $\mathrm{P}$ value (TS vs. controls) & MA & $P$ value (TS vs. MA) \\
\hline TFC CX $($ mean \pm SD $)$ & $25.48 \pm 6.1$ & $17.05 \pm 4.6$ & $<0.001$ & $28.79 \pm 10.33$ & 0.022 \\
\hline TFC RCA (mean \pm SD) & $26.43 \pm 8.95$ & $15.74 \pm 4.27$ & $<0.001$ & $25.83 \pm 11.57$ & 0.72 \\
\hline Average TFC (mean \pm SD) & $25.70 \pm 5.34$ & $16.7 \pm 3.26$ & $<0.001$ & $28.25 \pm 9.3$ & 0.046 \\
\hline
\end{tabular}

TFC, TIMI frame count; LAD, left anterior descending coronary artery; CX, circumflex artery; RCA, right coronary artery; TS, Takotsubo syndrome; MA, microvascular angina.

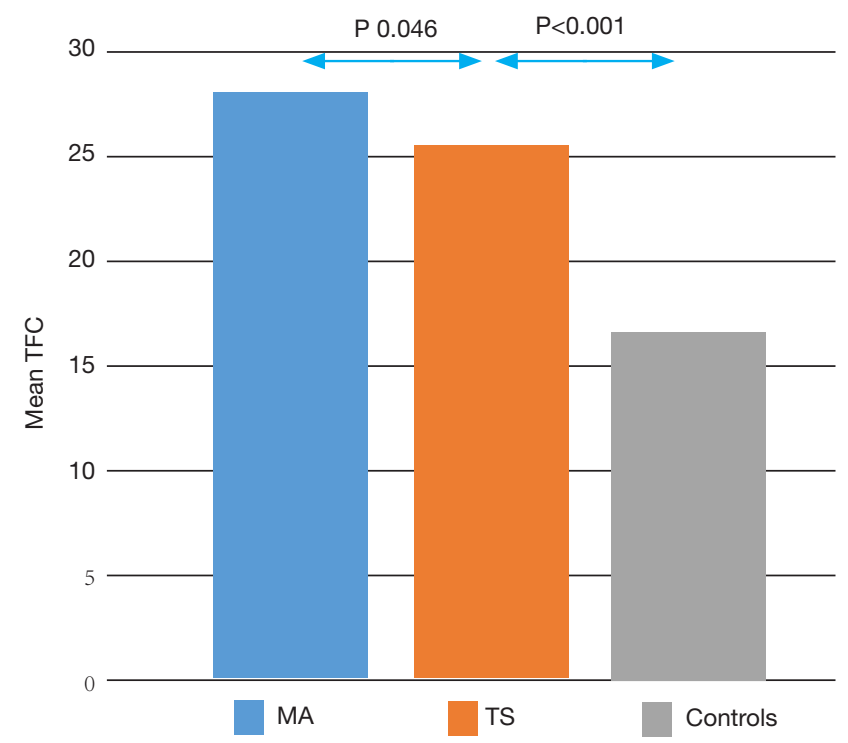

Figure 1 Mean TFC in TS, MA and controls. TFC, TIMI frame count; TS, Takotsubo syndrome; MA, microvascular angina.

MA. The extent of the alteration and the influence of cardiovascular risk factors, in the genesis of microcirculatory alterations was investigated.

Statistical analyses were performed using the MedCalc software. Numeric variables were expressed as mean plus or minus standard deviation (SD), categorical variables were expressed as a percentage. The differences between two numerical variables were analyzed by the ANOVA test, the differences between categorical variables, using the CHISQUARED test. It was considered a statistically significant a $\mathrm{P}$ value $<0.05$. ROC curves were drawn to determine which value of TFC had the best significance and specificity to differentiate normal individuals from patients with TS.

\section{Results}

We enrolled 71 consecutive patients with TS, 69 women $(97.18 \%)$ and 2 men $(2.82 \%)$, with a mean age of $65.27 \pm 9.53$ years, admitted to the Cardiology Unit of University Hospital Paolo Giaccone $(n=27)$, Cervello Hospital $(\mathrm{n}=19)$ and of Ingrassia Hospital of Palermo, Italy $(\mathrm{n}=25)$ from 2007 to 2014. Moreover we enrolled 70 controls, 34 women (48.57\%), and 36 men $(51.43 \%)$, with a mean age of $56.63 \pm 13.5$ years, without cardiovascular risk factors and coronary artery disease and 71 patients with MA, 69 women $(97.18 \%)$ and 2 men $(2.82 \%)$, with a mean age of $65.9 \pm 9.2$ admitted to the Cardiology Division of the University Hospital P. Giaccone. Clinical characteristics of the patients with TS are summarized in Table 1.

The average LVEF was $42 \% \pm 14.75 \%$ at admission and $59.5 \% \pm 0.13 \%$ at discharge. Wall motion analysis showed an apical ballooning in all the examined TS patients and there was no evidence of atypical variants. The mean troponin peak was $6.05 \pm 6.86 \mathrm{ng} / \mathrm{mL}$. In all patients, the coronary angiography excluded the presence of hemodynamically significant stenosis, occlusion and / or plaque rupture.

The assessment of the microcirculation, carried out through the TFC, showed significantly altered values in patients with TS compared with healthy controls. Comparing the average TFC for each vessel in patients with TS than in healthy controls, it was significantly higher in the former (LAD 25.16 \pm 6.91 vs. $17.30 \pm 3.76$, $\mathrm{P}<0.001$; CX 25.48 \pm 6.10 vs. 17.05 $\pm 4.60, \mathrm{P}<0.001$; RCA $26.43 \pm 8.95$ vs. $15.74 \pm 4.27, \mathrm{P}<0.001$. Table 2). The same result was confirmed by comparing the average TFC values found on all three vessels between patients with TS and controls (average TFC $25.70 \pm 5.34$ vs. 16.70 3.26 , $\mathrm{P}<0.001$, Figure 1, Table 2).

The ROC curve analysis showed that a TFC $>20$ frames is able to discriminate patients with TS from healthy controls with a specificity of $88.57 \%$ and sensitivity of 85.92\% (AUC 0.927, $\mathrm{P}<0.0001$, Figure 2). 


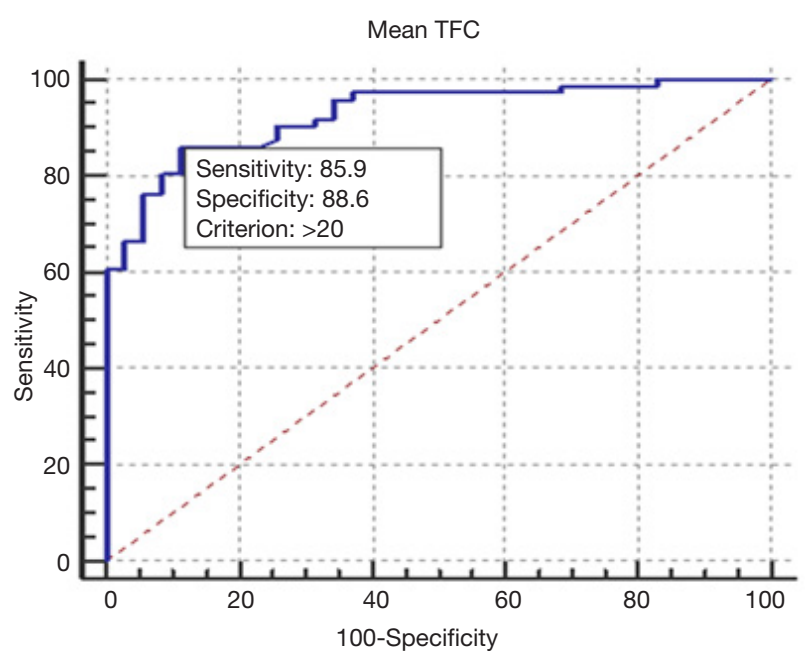

Figure 2 ROC curves: a mean TFC $>20$ differentiate between controls and TS. ROC, receiver operating characteristic; TFC, TIMI frame count; TS, Takotsubo syndrome.

The alteration of the microcirculation was diffuse in TS, in fact, there were no statistically significant differences in the comparison of TFC values between the three main vessels and it didn't coincide with the territory perfused by a single epicardial coronary artery, consistently with the extent of wall motion abnormalities evaluated by echocardiogram.

Specifically, a TFC value above $>20$ frames in all three vessels was found in $47 \%$ of patients $(n=33)$, while in $28 \%$ of patients $(n=20)$ impaired values were found simultaneously on two vessels and in $18 \%$ of cases $(n=13)$ in a single vessel. In 5 patients (7\%), we did not find values of TFC $>20$ frames in any vessel.

Considering as widespread a microcirculatory alteration in at least 2 vessels, we noticed that this was present in the $80.3 \%$ of patients with TS.

After confirming, by comparison with healthy controls, that a microvascular dysfunction is certainly demonstrable in patients with a diagnosis of TS, we compared the characteristics of microvascular impairment in TS and in MA. The mean TFC was slightly higher in patients with MA than in TS (TFC in MA $28.25 \pm 9.3$ vs. $25.7 \pm 5.34$ in TS, $\mathrm{P}<0.046$, Figure 1).

The alteration was diffuse, across the coronary bed, also in MA patients, as shown in Table 2.

A TFC value above 20 frames in all three vessels was found in $57.74 \%$ of patients $(n=41)$, while in $28.17 \%$ of patients $(n=20)$ impaired values were found simultaneously on two vessels and in $7.04 \%$ of cases $(n=5)$ in a single vessel. In 5 patients $(7.04 \%)$, we did not find values of TFC $>20$ frames in any vessel. Figure 3 shows the distribution of TFC $>20$ frames across vessels in MA and TS.

The prevalence of cardiovascular risk factors in TS and MA was not significantly different except for obesity, that is known to be strictly associated with MA (Figure 4). At multivariate analysis, the variables that were significantly associated with a TFC impairment were TS (OR 6.21, IC 95\%: 1.2-32.1, $\mathrm{P}=0.029)$ and arterial hypertension (OR 9.38, IC 95\%: 2.33-37.77, $\mathrm{P}=0.0016$ ).

We found a linear relation between EF and average TCF in TS (Figure 5).

\section{Discussion}

The pathophysiology of TS is incompletely understood, and microvascular dysfunction is suggested as a pathogenetic hypothesis. Several studies have demonstrated the presence of impaired coronary microcirculation in patients with TS using different diagnostic tools such as angiography, nuclear cardiology, and echocardiography, however, the exact mechanism by which coronary microvascular dysfunction occurs remain unclear and particularly it is unclear if it is a causative factor or a bystander.

Some studies recorded alterations of the coronary microcirculation, at TFC analysis observing the coronary angiograms during the acute phases of the TS (13-18). Kume et al. detected the dysfunction of the coronary microcirculation with Doppler flow-wire technique in patients with TS (19). Other authors demonstrated, in patients with TS, abnormalities of the microcirculation through myocardial perfusion scintigraphy with technetium $99 \mathrm{~m}$ tetrofosmin (20-22). A disorder of glucose and fatty acid metabolism, even more, severe of the perfusion defect, has also been demonstrated (23-24). Abnormalities of coronary microcirculation have been also detected using contrast echocardiography (25-26). In accordance with angiographic studies, these authors demonstrated that they occur in the acute phase and typically recover at the resolution of the syndrome, even before wall motion abnormalities.

Most of the studies compared the characteristics of microcirculatory impairment in TS with acute coronary syndromes and healthy controls. They showed that the impairment of microvascular perfusion in TS is usually diffuse and not limited to the territory of a single epicardial 


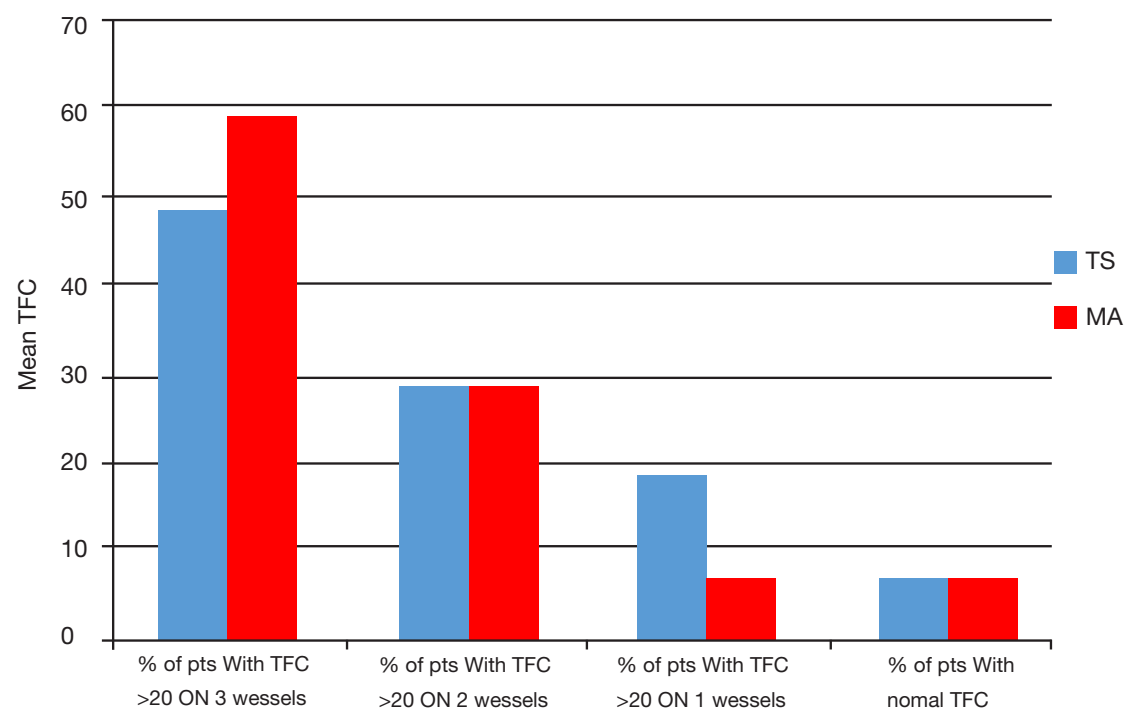

Figure 3 Distribution of TFC value above $>20$ frames across vessels $(3,2,1,0)$ in TS and MA. TFC, TIMI frame count; TS, Takotsubo syndrome; MA, microvascular angina.

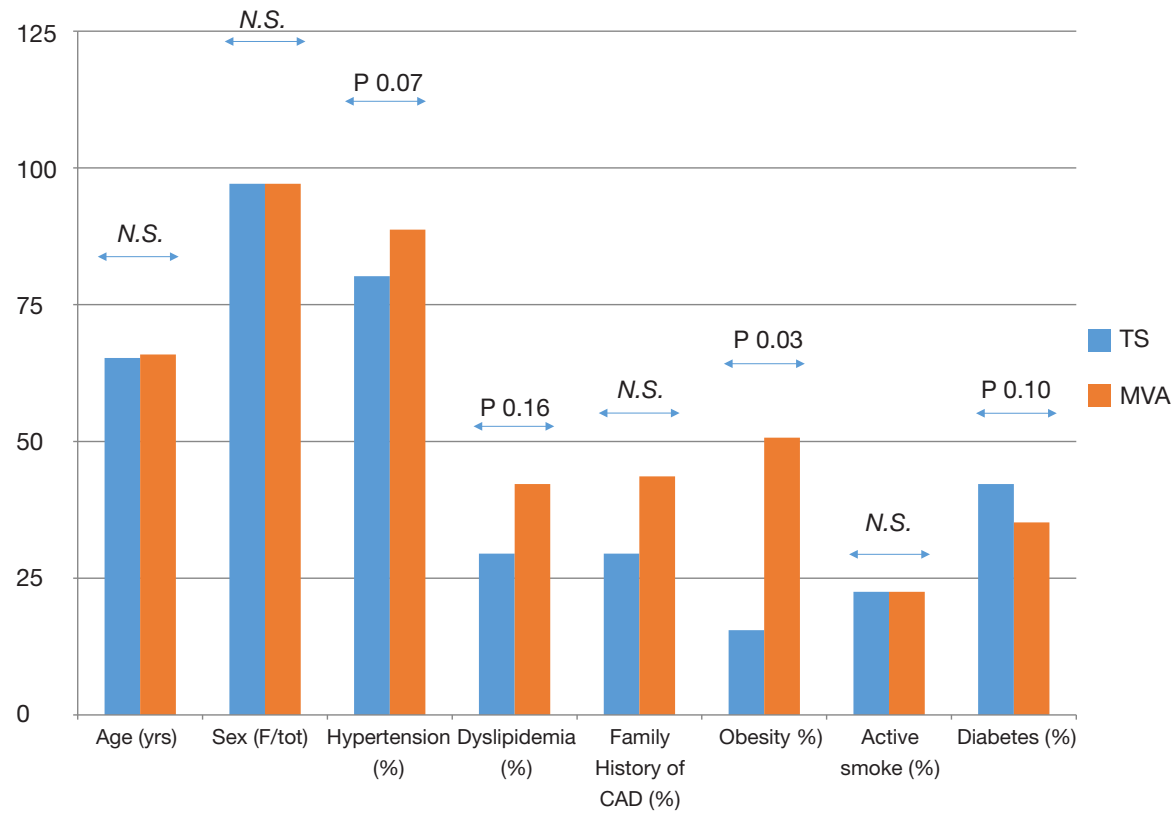

Figure 4 Prevalence of risk factors in TS and MA. TS, Takotsubo syndrome; MA, microvascular angina.

coronary artery (13), microcirculation is more impaired in patients with TS than in controls and in patients with STEMI exhibiting myocardial reperfusion, while it is less impaired than in patients with STEMI exhibiting microvascular occlusion (27).

According to our knowledge, this is the first study that compared the characteristics of microcirculation in patients with TS and with MA. In a previous report, Takahashi et al. described the occurrence of TS in patients with preexisting MA and they postulated that it could predispose to TS and it accentuates during its course (28). In contrast with this hypothesis, it must be pointed out that in most 


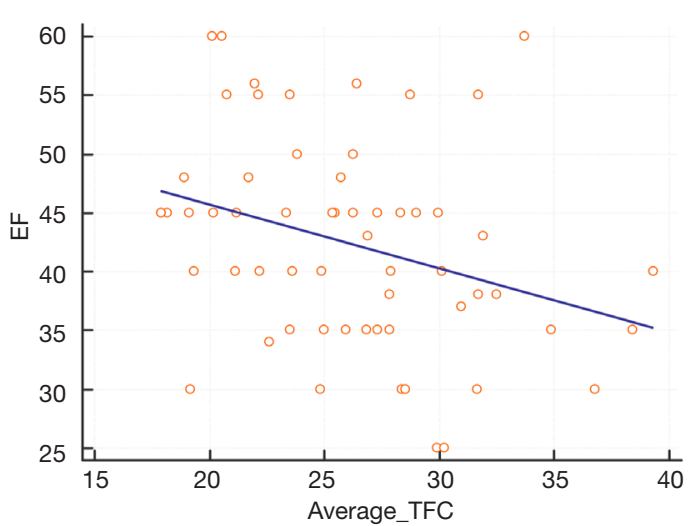

Figure 5 Scatter plot showing the relationship between EF and mean TFC. EF, ejection fraction; TFC, TIMI frame count.

of the cases TS occurs in old women without any previous history of MA and that it has been demonstrated the microvascular impairment recovers after the resolution of the syndrome $(25,26)$.

Accordingly, none of our patients had a history of MA preceding TS. The prevalence of cardiovascular risk factors, except for obesity, that was more frequent in MA, was not different is TS and MA, and for this reason, the microvascular dysfunction is probably proper of the TS and not secondary to the presence of cardiovascular risk factors.

The coronary microcirculation dysfunction in TS was found to be mild and wide spread, usually involving at least two vessels.

We detected cut-off values ( $>20$ frames) to discriminate between patients with normal microcirculation and patients with TS. The microcirculatory abnormalities in TS were milder than those observed in patients with MA; we did not find a cut-off value able to discriminate between MA and TS.

The main limitation of our study is that we used to assess microcirculatory function indirect methods that are less accurate in defining microcirculatory impairment compared with other methods such as PET, MRI or contrast echocardiography, that unfortunately, it was not possible to perform. It is advisable to use these more precise techniques in future studies to better define microvascular involvement in TS compared to MA.

\section{Conclusions}

Our study confirms that in patients with TS there is a microvascular impairment, detectable by TFC. This dysfunction is mild and a cut off value of TFC $>20$ frames has high sensitivity and specificity in discriminating patients with TS from controls. We demonstrated that microvascular abnormalities are usually widespread, as well as in patients with MA, and not involving the territory of a single epicardial coronary artery, in accordance with the extension of wall motion abnormalities. Compared with MA, the amount of microvascular dysfunction is slightly lower.

\section{Acknowledgements}

None.

\section{Footnote}

Conflicts of Interest: The authors have no conflicts of interest to declare.

Ethical Statement: The ethic committee approval was not requested because the study was observational and retrospective, it did not subjected patients to additional risks and it did not modify the normal clinical practice. Every patients signed informed consent for the use of their clinical data.

\section{References}

1. Novo S, Akashi Y, Arbustini E, et al. Takotsubo cardiomyopathy: a consensus document. G Ital Cardiol (Rome) 2008;9:785-97.

2. Maron BJ, Towbin JA, Thiene G, et al. Contemporary definitions and classification of the cardiomyopathies: an American Heart Association Scientific Statement from the Council on Clinical Cardiology, Heart Failure and Transplantation Committee; Quality of Care and Outcomes Research and Functional Genomics and Translational Biology Interdisciplinary Working Groups; and Council on Epidemiology and Prevention. Circulation 2006;113:1807-16.

3. Akashi YJ, Goldstein DS, Barbaro G, et al. Takotsubo cardiomyopathy: a new form of acute, reversible heart failure. Circulation 2008;118:2754-62.

4. Tsuchihashi K, Ueshima K, Uchida T, et al. Transient left ventricular apical ballooning without coronary artery stenosis: a novel heart syndrome mimicking acute myocardial infarction. Angina Pectoris-Myocardial Infarction Investigations in Japan. J Am Coll Cardiol 2001;38:11-8.

5. Kida K, Akashi YJ, Fazio G, et al. Takotsubo 
cardiomyopathy. Curr Pharm Des 2010;16:2910-7.

6. Lyon AR, Bossone E, Schneider B, et al. Current state of knowledge on Takotsubo syndrome: a position statement from the taskforce on Takotsubo syndrome of the heart failure association of the European society of cardiology. Eur J Heart Fail 2016;18:8-27.

7. Sato H, Tateishi H, Uchida T, et al. Takotsubo-type cardiomyopathy due to multivessel spasm. In: Kodama K, Haze K, Hon M, eds. Clinical aspects of myocardial injury: from ischemia to heart failure. Tokyo: Kagakuhyouronsha, 1990:56-64.

8. Sharkey SW, Lesser JR, Zenovich AG, et al. Acute and reversible cardiomyopathy provoked by stress in women from the United States. Circulation 2005;111:472-9.

9. Assennato P, Alfano R, Novo G, et al. Two cases of tako-tsubo cardiomyopathy in Caucasians. Ital Heart J 2005;6:614-7.

10. Desmet WJ, Adriaenssens BF, Dens JA. Apical ballooning of the left ventricle: first series in white patients. Heart 2003;89:1027-31.

11. Prasad A, Lerman A, Rihal CS. Apical ballooning syndrome (Tako-Tsubo or stress cardiomyopathy): a mimic of acute myocardial infarction. Am Heart J 2008;155:408-17.

12. Gibson CM, Cannon CP, Daley WL, et al. TIMI frame count: a quantitative method of assessing coronary artery flow. Circulation 1996;93:879-88.

13. Fazio G, Sarullo FM, Novo G, et al. Tako-Tsubo cardiomyopathy and microcirculation. J Clin Monit Comput 2010;24:101-5.

14. Elesber A, Lerman A, Bybee KA, et al. Myocardial perfusion in apical ballooning syndrome correlate of myocardial injury. Am Heart J 2006;152:469.e9-13.

15. Khalid N, Iqbal I, Coram R, et al. Thrombolysis in myocardial infarction frame count in Takotsubo cardiomyopathy. Int J Cardiol 2015;191:107-8.

16. Khalid N, Iqbal I, Ikram S. Thrombolysis in myocardial infarction frame count in Takotsubo cardiomyopathy. J Am Coll Cardiol 2013;61:E50.

17. Bybee KA, Prasad A, Barsness GW, et al. Clinical characteristics and thrombolysis in myocardial infarction frame counts in women with transient left ventricular apical ballooning syndrome. Am J Cardiol 2004;94:343.

18. Sharkey SW, Lesser JR, Menon M, et al. Spectrum and significance of electrocardiographic patterns, troponin levels, and thrombolysis in myocardial infarction frame count in patients with stress (tako-tsubo) cardiomyopathy and comparison to those in patients with ST-elevation anterior wall myocardial infarction. Am J Cardiol 2008;101:1723-8.

19. Kume T, Akasaka T, Kawamoto T, et al. Assessment of coronary microcirculation in patients with Takotsubo-like left ventricular dysfunction. Circ J 2005;69:934-9.

20. Abe Y, Kondo M, Matsuoka R, et al. Assessment of clinical features in transient left ventricular apical ballooning. J Am Coll Cardiol 2003;41:737-42.

21. Nishikawa S, Ito K, Adachi Y, et al. Ampulla ('Takotsubo') cardiomyopathy of both ventricles: evaluation of microcirculation disturbance using $99 \mathrm{~m}$ Tc-tetrofosmin myocardial single photon emission computed tomography and doppler guide wire. Circ J 2004;68:1076-80.

22. Ito K, Sugihara H, Katoh S, et al. Assessment of Takotsubo (ampulla) cardiomyopathy using 99mTc-tetrofosmin myocardial SPECT--comparison with acute coronary syndrome. Ann Nucl Med 2003;17:115-22.

23. Yoshida T, Hibino T, Kako N, et al. A pathophysiologic study of Takotsubo cardiomyopathy with F-18 fluorodeoxyglucose positron emission tomography. Eur Heart J 2007;28:2598-604.

24. Kurisu S, Inoue I, Kawagoe T, et al. Myocardial perfusion and fatty acid metabolism in patients with tako-tsubolike left ventricular dysfunction. J Am Coll Cardiol 2003;41:743-8.

25. Galiuto L, De Caterina A, Porfidia A, et al. Reversible coronary microvascular dysfunction: a common pathogenetic mechanism in Apical Ballooning or TakoTsubo Syndrome. Eur Heart J 2010;31:1319-27.

26. Jain M, Upadaya S, Zarich SW. Serial evaluation of microcirculatory dysfunction in patients with Takotsubo cardiomyopathy by myocardial contrast echocardiography. Clin Cardiol 2013;36:531-4.

27. De Caterina AR, Leone AM, Galiuto L, et al. Angiographic assessment of myocardial perfusion in TakoTsubo syndrome. Int J Cardiol 2013;168:4717-22.

28. Takahashi H, Tani S, Kikushima K, et al. Takotsubo cardiomyopathy in two patients with microvascular angina. J Cardiol Cases 2015;12:26-9.

Cite this article as: Nove G, Quagliana A, Buccheri D, Rizzo S, Giambanco S, Giambanco F, Evola S, Geraci G, Novo S. Characteristics of coronary microcirculatory function in patients with Takotsubo syndrome. J Thorac Dis 2017;9(11):4531-4537. doi: $10.21037 /$ jtd.2017.10.93 